\title{
Evaluation of Fibrosis Progression by Noninvasive Biochemical Markers in Egyptian Patients with Chronic Hepatitis B
}

\author{
${ }^{1}$ Tarek A Besheer, ${ }^{1}$ Mahmoud M El-Bendary, ${ }^{2}$ Khaled R Zalata, ${ }^{3}$ Hossam E Zaghalol \\ ${ }^{1}$ Department of Tropical Medicine, Faculty of Medicine, Mansoura University, Mansoura, Egypt \\ ${ }^{2}$ Department of Pathology, Faculty of Medicine, Mansoura University, Mansoura, Egypt \\ ${ }^{3}$ Department of Clinical Pathology, Faculty of Medicine, Mansoura University, Mansoura, Egypt
}

Correspondence: Tarek A Besheer, Lecturer, Department of Tropical Medicine, Faculty of Medicine, Mansoura University Mansoura-35516, Egypt, Phone: +20507759699, +20127977075, e-mail: tarekbesheer@yahoo.com

\section{ABSTRACT}

Background: To evaluate the usefulness of biochemical markers for prediction of fibrosis in chronic hepatitis $\mathrm{B}$.

Materials and methods: This study was carried out on 133 patients with chronic hepatitis $B$. The diagnostic accuracy of four noninvasive liver fibrosis markers; matrix metalloproteinase-2 (MMP-2), hyaluronic acid (HA), aspartate aminotransferase (AST) to platelet ratio index (APRI) and FIB-4 was evaluated.

Results: The levels of HA and MMP-2 were increased along with the severity of fibrosis. The cutoff value for HA was $265 \mathrm{ng} / \mathrm{ml}$ for predicting liver cirrhosis, whereas cutoff value for MMP-2 was $860 \mathrm{ng} / \mathrm{ml}$. The cutoff value for APRI for detecting severe fibrosis (F3 and F4) was $\geq 0.9$. Finally, cutoff value for FIB-4 was $\geq 0.9$ for assessment of severe fibrosis in chronic hepatitis B.

Conclusion: HA, MMP-2, APRI and FIB-4 could be clinically useful as noninvasive markers for detection of severe fibrosis in chronic hepatitis B. Abbreviations: HBV: Hepatitis B virus; HCV: Hepatitis C virus; HCC: Hepatocellular carcinoma; $\mathrm{CHB}$ : Chronic hepatitis B; HDV: Hepatitis delta virus; HBsAg: Hepatitis B surface antigen; ALT: Alanine aminotransferase; AST: Aspartate aminotransferase; APRI: AST to platelet ratio index; PLT: Platelet; ULN: Upper limit of normal; HA: Hyaluronic acid; MMP-2: Matrix metalloproteinase-2; ROC: Receiver operating curve.

Keywords: APRI, CHB, FIB-4, HA, Hepatic fibrosis, MMP-2.

\section{INTRODUCTION}

Hepatitis B virus (HBV) and hepatitis C virus (HCV), the major causes of chronic liver diseases, are responsible for hepatic inflammation, liver fibrosis, liver cirrhosis and hepatocellular carcinoma (HCC) ${ }^{1-3}$ Several recent progresses have been accomplished in the diagnosis and treatment of chronic liver diseases. However, it seems that conventional biochemical and serological tests are of limited value in the diagnosis of the degree of fibrosis. ${ }^{4}$ Liver biopsy is currently the gold standard of determining the severity of necroinflammation and staging of fibrosis, ${ }^{5,6}$ but it has been well-documented that complications like pain, bleeding and rarely death may occur during liver biopsy. In addition, interand intraobserver error may lead to incorrect staging of up to $33 \%$ of biopsies. ${ }^{7}$ In addition, the metavir staging system may not reflect a linear increase in fibrosis. In particular, the increase in the degree of fibrosis between F1 (enlarged portal tract) and F2 (enlarged portal tract with rare septae) may not be as great as the increase between F2 and F3 (enlarged portal tract with numerous septae). Indeed, in early-stage disease, there is poor correlation between degree of liver fibrosis as detected by digital image analysis and staging by a pathologist. ${ }^{8}$ The appropriateness of repeating biopsy is increasingly questionable; accordingly, accurate noninvasive markers have been now validated. ${ }^{7-9}$ Liver biopsy cannot be performed universally in all patients with impaired homeostasis of any cause. ${ }^{10}$

Hence, noninvasive markers for the prediction of liver fibrosis and cirrhosis become essential. Therefore, there is a growing tendency to use noninvasive measures instead of histopathological analysis of liver tissue for the evaluation of disease progression in patients with chronic liver diseases. ${ }^{11}$ Because serum markers are likely to reflect the quantity of fibrotic matrix/tissue, they may correlate better with fibrosis as detected by image analysis than stage as determined by a pathologist. Up to date, several laboratory tests, scores and indices have been proposed for noninvasive prediction of hepatic fibrosis in chronic hepatitis B (CHB) patients. ${ }^{11,12}$ In this study, we evaluated the clinical usefulness of biochemical markers for invasive diagnosis of fibrosis in Egyptian patients with CHB.

\section{MATERIALS AND METHODS}

\section{Patients}

Study was carried out on 133 patients with CHB and 35 healthy controls. The study was conducted in accordance 
with the declaration of Helsinki, and all patients gave informed consent. Exclusion criteria were chronic liver disease due to other causes or coinfection with hepatitis $C$ virus or HCV, previous or concomitant anti-HBV therapy. Chronic HBV infection was diagnosed based on positive hepatitis B surface antigen (HBsAg) and levels of alanine aminotransferase (ALT). Full blood counts including platelet counts (PLT), prothrombin time, liver function tests (ALT, aspartate aminotransferase (AST), alkaline phosphatase and total bilirubin), HBsAg and anti-HCV Ab were evaluated by commercially available assays. APRI was calculated by the equation: [(AST/ULN, upper limit of normal $) \times 100] /$ PLT $\left.\left(10^{9} / \mathrm{L}\right)\right] .{ }^{13}$ FIB-4 was estimated from the formula; FIB-4 $=$ [age $(\mathrm{yr}) \times$ AST $(\mathrm{U} / \mathrm{L})] /\left\{\left[\mathrm{PLT}\left(10^{9} /\right.\right.\right.$ $\mathrm{L})] \times(\operatorname{ALT}(\mathrm{U} / \mathrm{L})] 1 / 2\} .{ }^{14} \mathrm{HA}$ was estimated by enzymelinked immunosorbent assay (Corgenix Inc. CO, USA) and MMP-2 by Amersham Biosciences (GE Healthcare, Filial Sverige, Björkgatan, Uppsala, Sweden).

Liver biopsies were obtained by ultrasound-guided techniques. Liver specimens were fixed, paraffin-embedded, and stained with hematoxylin and eosin. Biopsy specimens with at least four portal fields were considered representative and scored by a pathologist. Fibrosis was staged as no (0), mild (1), moderate (2), severe (3) and cirrhosis (4), using the metavir score. Hepatic inflammatory activity was also scored. ${ }^{15}$

\section{Statistical Analysis}

Statistical analysis was done by using SPSS program version 10. The data were parametric by using Kolmograv-Smirnov test. The qualitative data were presented in the form of number and percentage. Chi-square test was used for qualitative data. The quantitative data were presented in the form of mean, standard deviation and range. Student t-test was used for comparison of two groups. Pearson correlation coefficient was used to study the relation between each two items in every group. ROC (receiver operating curve) was done to determine a cutoff point; sensitivity and specificity were calculated for this cutoff point. Significance was considered when p-value less than 0.05 .

\section{RESULTS}

\section{Patient Characteristics}

Table 1 has shown the baseline characteristics of all patients. The age of the patients was $45.8 \pm 9.1$ years. The numbers of male were $84(63.16 \%)$ and 49 patients were female (36.84\%). No or mild liver fibrosis (stages F0, F1 and F2) was observed in 38 patients. Severe liver fibrosis of stage F3 and F4 was detected in 46 and 49 patients, respectively.
Taken together, severe degrees of liver fibrosis (F3 and F4) were detected in a total of 95 patients. Almost no or minimal grades of inflammation were seen in 41 patients (30.8\%), whereas, severe inflammation (A3 and A4) was seen in 92 (69.2\%) patients.

\section{Correlation between Noninvasive Markers and Fibrosis Stage}

To assess the clinical implications of noninvasive markers of liver fibrosis we checked the levels of serum HA, MMP2, APRI and FIB-4 in patients with CHB (Table 2). AUROC was used to evaluate the overall diagnostic performance of scores (Figs 1A to D). The levels of HA were $135 \pm$ $30,230 \pm 62$, and $296 \pm 150 \mathrm{ng} / \mathrm{ml}$ in patients with minimal fibrosis (fibrosis F0+F1+F2), fibrosis level of F3 and fibrosis level of F4, respectively. The levels of MMP-2 were $625 \pm$ $57,789 \pm 85$, and $1165 \pm 89 \mathrm{ng} / \mathrm{ml}$ in patients with minimal fibrosis (fibrosis F0+F1+F2), fibrosis level of F3 and fibrosis level of F4, respectively. Thus, the serum levels of HA and MMP-2 showed a significant upward tendency as the levels of fibrosis became severe. The cutoff value for HA was $265 \mathrm{ng} / \mathrm{ml}$ for severe fibrosis of $\mathrm{F} 4$ (according to AUROC curve at which sensitivity was $83 \%$, specificity was $89.2 \%$ ) (Fig. 1). The cutoff value for MMP-2 was $860 \mathrm{ng} / \mathrm{ml}$ with a sensitivity $84 \%$ and specificity $87.1 \%$. HA was increased with the severity grades of liver inflammation (minimal, mild inflammation and moderate inflammation $)(\mathrm{p}<0.001)$ whereas this trend was not seen in MMP-2 ( $p=0.21)$.

The mean APRI was $\geq 1.32$ in patients with severe fibrosis (F3 and F4) and $<0.72$ in patients with no or mild to moderate fibrosis (F0, F1 and F2). The APRI showed a significant correlation with advanced liver fibrosis $(\mathrm{p}<0.05)$ with the cutoff value for detecting severe fibrosis (F3 and F4) was $\geq 0.9$ according to AUROC curve at which sensitivity was $71 \%$ and the specificity was $76.3 \%$.

The mean FIB- 4 was $\geq 1.47$ in patients with severe fibrosis (F3 and F4) and $<0.61$ in patients with no or mild to moderate fibrosis (F0, F1 and F2). The FIB-4 showed a significant correlation with advanced liver fibrosis $(\mathrm{p}<0.05)$ with the cutoff value for detecting severe fibrosis (F3 and F4) was $\geq 0.9$ according to AUROC curve with a sensitivity of $73.4 \%$ and specificity of $78.7 \%$.

Correlation between different noninvasive serum markers has been shown in Table 3 .

\section{DISCUSSION}

In HBV-infected patients, noninvasive methods of assessment of hepatic fibrosis are not as well validated as in patients with CHC. Furthermore, the exact staging of liver fibrosis is crucial for the therapeutic decision and 

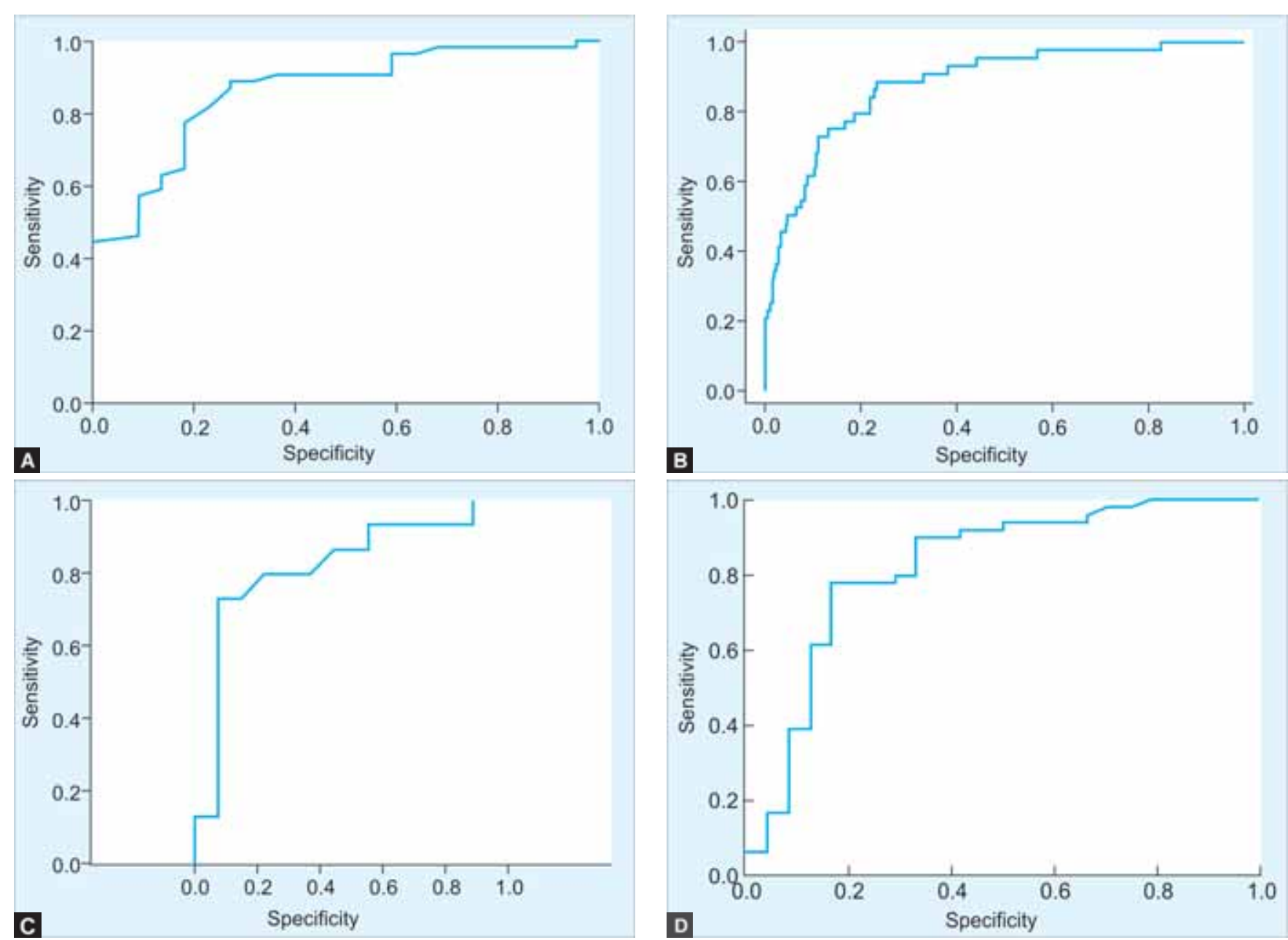

Figs 1A to D: (A) ROC curve for HA which showed that cutoff value for HA was $265 \mathrm{ng} / \mathrm{ml}$ could predict cirrhosis (F4) according to AUROC curve with a sensitivity $83 \%$ and specificity $89.2 \%$, (B) ROC curve for MMP-2 which showed that cutoff value for MMP-2 was 860 $\mathrm{ng} / \mathrm{ml}$ could predict cirrhosis (F4) according to AUROC curve with sensitivity of $84 \%$ and specificity of $87.1 \%$, (C) ROC curve for APRI which showed that cutoff value of APRI was set at 0.9 for detecting severe fibrosis (F3 and F4) with a sensitivity of $71 \%$ and specificity of $76.3 \%$ in CHB patients, (D) ROC curve for FIB-4 cutoff value of FIB-4 was set at 0.9 for detecting severe fibrosis (F3 and F4) at sensitivity $73.4 \%$ and specificity $78.7 \%$ in $\mathrm{CHB}$ patients

assessing the prognosis of CHB patients. ${ }^{11,16,17}$ Conversely, in HBV inactive carriers or immunotolerant patients, in whom liver biopsy is not indicated, noninvasive methods could be useful to strengthen the diagnosis by confirming the absence of significant fibrosis. Conventional biochemical and serological tests are of little value in the diagnosis of the degree of fibrosis and the activity of fibrogenesis, and percutaneous liver biopsy is therefore used to assess the extent of liver fibrosis and fibrogenesis. ${ }^{4,11}$ However, a liver biopsy is sometimes of questionable value because of the heterogeneous distribution of pathological changes in the liver. For instance, even a $25 \mathrm{~mm}$ long liver biopsy has a $25 \%$ rate of discordance for fibrosis staging. ${ }^{18}$ Also, when the specimen size is adequate, the level of experience (specialization, duration and location of practice) of the pathologist may even be more important. As a result, noninvasive biochemical markers for assessing liver fibrosis in chronic hepatitis are being actively sought to help evaluate histologic damage and monitor the progression of fibrosis. ${ }^{11}$
In this study, noninvasive markers are mainly based on two kinds of serum markers, direct and indirect. Direct serum markers are directly linked to the modifications in extracellular matrix (ECM) metabolism (HA and MMP-2). Indirect serum markers have no direct link with liver fibrosis but reflect liver dysfunction or other phenomena caused by fibrosis (APRI and FIB-4). ${ }^{14}$ The present study showed that HA was significantly elevated in CHB and patients with increased fibrosis than the control group ( $<<0.001)$. HA was linearly increased significantly with the different stages of fibrosis $(p<0.001)$ and the highest concentration being seen in patients with fibrosis of F4. Cutoff value for HA was $265 \mathrm{ng} / \mathrm{ml}$ could predict cirrhosis (F4) according to AUROC curve with a sensitivity $83 \%$ and specificity $89.2 \%$. This was in accordance with Murawaki et al and Xu et al, ${ }^{19,20}$ who reported that serum HA levels correlated with degree of fibrosis and Child-Pugh score. This may be explained by the impaired elimination of HA by the sinusoidal 
Table 1: Clinical characteristics of patients with chronic hepatitis $\mathbf{B}(\mathrm{CHB})$

\begin{tabular}{lc} 
Patient characteristics & CHB patient \\
\hline Age (years) & $45.8 \pm 9.1$ \\
\hline Sex & Number $(\%)$ \\
Male & $84(63.16 \%)$ \\
Female & $49(36.84 \%)$ \\
\hline Stage of fibrosis & Number $(\%)$ \\
F0 + F1 + F2 & $38(28.57 \%)$ \\
F3 & $46(34.59 \%)$ \\
F4 & $49(36.84 \%)$ \\
\hline Grade of inflammation & Number $(\%)$ \\
A0 + A1 + A2 & $41(30.8 \%)$ \\
A3 & $52(39.1 \%)$ \\
A4 & $40(30.1 \%)$ \\
\hline Platelet counts $(\times 1000 / c u ~ m m)$ & $158.3 \pm 76.3$ \\
INR & $1.21 \pm 0.13$ \\
AST (U/L) & $56.9 \pm 39.2$ \\
ALT (U/L) & $64.3 \pm 42.5$ \\
Alkaline phosphatase (mg/dl) & $132.4 \pm 59.6$ \\
Total bilirubin (mg/dl) & $0.7 \pm 0.4$ \\
\hline
\end{tabular}

Table 2: Comparison of noninvasive serum markers among different stages of liver fibrosis in patients with chronic hepatitis $B(C H B)$

\begin{tabular}{ll} 
Patient characteristics & CHB (mean \pm SD) \\
\hline HA (ng/ml) & \\
F0 + F1 + F2 & $185 \pm 30$ \\
F3 & $230 \pm 62$ \\
F4 & $296 \pm 150$ \\
\hline MMP-2 (ng/ml) & \\
F0 + F1 + F2 & $625 \pm 57$ \\
F3 & $789 \pm 85$ \\
F4 & $1165 \pm 89$ \\
\hline APRI & \\
F0 + F1 + F2 & $0.51 \pm 0.24$ \\
F3 & $0.72 \pm 0.21$ \\
F4 & $1.91 \pm 0.35$ \\
\hline FIB-4 & \\
F0 +F1 + F2 & $0.61 \pm 0.12$ \\
F3 & $0.82 \pm 0.51$ \\
F4 & $2.12 \pm 0.24$ \\
\hline
\end{tabular}

Table 3: Correlation between noninvasive serum markers in all patients

\begin{tabular}{lrr} 
HA and MMP-2 & 0.63 & $<0.001$ \\
HA and APRI & 0.71 & 0.002 \\
HA and FIB-4 & 0.27 & 0.018 \\
MMP-2 and APRI & 0.33 & 0.004 \\
MMP-2 and FIB-4 & 0.45 & $<0.001$ \\
APRI and FIB-4 & 0.64 & 0.006 \\
\hline
\end{tabular}

capillarization and by the formation of basement membrane, in addition to the increased production of HA within the fibrotic liver, which occurs together with the accelerated synthesis of extracellular matrix. ${ }^{21}$ Guéchot et $\mathrm{al}^{22}$ showed that the sensitivity and specificity of serum HA at a cutoff point of $110 \mu \mathrm{g} / \mathrm{L}$ for the diagnosis of hepatic fibrosis are $79 \%$ and $89 \%$, respectively. Patel et $\mathrm{al}^{23}$ have reported that as a noninvasive valuable marker, serum HA concentration is correlated with hepatic fibrosis. Montazeri et al ${ }^{24}$ demonstrated that serum HA is a preferred marker of severe fibrogenesis and inflammation in CHB patients. HA at a cutoff point of $126.4 \mu \mathrm{g} / \mathrm{L}$ can detect severe fibrosis with a sensitivity of $90.9 \%$ and a specificity of $98.1 \%$. This difference in cutoff point could be due to the relatively smaller number of patients in this study, or the difference in the studied subjects regarding the Egyptian population (genetic and environmental factors or individual variation).

MMP-2 exhibits degradative activity against basementmembrane collagen, its release by activated hepatic stellate cells in the space of Disse disrupts the normal subendothelial liver matrix. Enhanced production of abnormal interstitial collagens (type I and II) subsequently leads to an abnormal basement-membrane collagen, which in turn, disrupts hepatocellular function and may lead to further stellate cell activation. The net effect of this activity seems to accelerate the replacement of the normal subendothelial matrix. ${ }^{25}$ MMP-2 showed a significant difference between different stages of fibrosis ( $p<0.001$ ). Cutoff value for MMP-2 was $860 \mathrm{ng} / \mathrm{ml}$ with sensitivity of $84 \%$ and specificity of $87.1 \%$. This was in agreement with Liu et $\mathrm{al}^{26}$ who reported that TIMP-2 was well correlated with the levels of liver cirrhosis indexes. However, this contrasted with Walsh et $\mathrm{al}^{27}$ and Boeker et $\mathrm{al}^{28}$ who reported that there was no correlation between fibrosis and levels of MMP-2. This difference could be attributed to the nature of patients as they studied only patients with $\mathrm{CHC}^{27}$

The cutoff value of APRI was set at 0.9 for detecting severe fibrosis (F3 and F4) with a sensitivity of 71\% and specificity of $76.3 \%$ in CHB patients. The sensitivity, specificity was high for the detection of severe fibrosis stages in CHB patients.

The cutoff value of FIB-4 was set at 0.9 for detecting severe fibrosis (F3 and F4) at sensitivity $73.4 \%$ and specificity $78.7 \%$ in $\mathrm{CHB}$ patients. The sensitivity, specificity was high for the detection of severe fibrosis stages in CHB patients. Zhang et $\mathrm{al}^{29}$ reported that both APRI and FIB-4 do not involve a complicated formula, thus allowing it to be quickly calculated. In addition, it uses two laboratory tests and is not associated with the added expense of a reference laboratory and does not contain subjective parameter. The possibility for the variation of platelet count 
in patients with chronic liver disease was due to many factors, such as platelet mean lifetime, thrombopoietin production, myelotoxic effects, virus types, different pathogenesis of liver fibrosis and sepsis. ${ }^{30}$ In addition, the AST level was significantly increased in the patients with liver fibrosis of F4 compared with the patients with stage F3 fibrosis, but there was no significant correlation in the prediction of liver fibrosis or cirrhosis. The elevation of AST might be due to the reduction in the clearance of AST and mitochondrial injury. ${ }^{31}$ The performance of any surrogates is classically evaluated by calculation of the area under the receiver operating characteristic curve (AUROC) using liver biopsy as the reference standard. Because liver biopsy is an imperfect gold standard, a perfect surrogate will never reach the maximal value (1.0). ${ }^{32}$ Taking into account a range of accuracies of the biopsy and a range of prevalence of significant disease (that influence the AUROC), Mehta et $\mathrm{al}^{33}$ have shown that in the most favorable scenario, an AUROC > 0.90 cannot be achieved even for a perfect marker.

\section{CONCLUSION}

HA, MMP-2, APRI and FIB-4 parameters potentially could be clinically useful as noninvasive markers for detection of severe fibrosis (F3 and F4) and decrease the number of liver biopsies in CHB patients.

\section{REFERENCES}

1. Sandrin L, Tanter M, Gennisson JL, Catheline S, Fink M. Shear elasticity probe for soft tissues with 1-D transient elastography. IEEE Trans Ultrason Ferroelectr Freq Control 2002;49:436-46.

2. Jacobson IM. Therapeutic options for chronic hepatitis B: Considerations and controversies. Am J Gastroenterol 2006;101:S13-S18.

3. Wong SN, Lok AS. Update on viral hepatitis. Curr Opin Gastroenterol 2006;22:241-47.

4. Friedman SL. Hepatic fibrosis. Schiff ER, Sorrell MF, Maddrey WC (Eds). In: Schiff's diseases of the liver (10th ed). Philadelphia, New York 2006;371-83.

5. Bravo AA, Sheth SG, Chopra S. Liver biopsy. N Engl J Med 2001;344:495-500.

6. Narasimhan G, Sargios TN, Kalakuntla R, et al. Treatment rates in patients with chronic hepatitis $\mathrm{C}$ after liver biopsy. J Viral Hepat 2006;13:783-86.

7. Sebastiani G, Alberti A. Noninvasive fibrosis biomarkers reduce but not substitute the need for liver biopsy. World J Gastroenterol 2006;12:3682-94.

8. O’Brien MJ, Keating NM, Elderiny S, et al. An assessment of digital image analysis to measure fibrosis in liver biopsy specimens of patients with chronic hepatitis C. Am J Clin Pathol 2000;114:712-18.

9. Poynard T, Morra R, Halfon P, et al. Meta-analyses of fibrotest diagnostic value in chronic liver disease BMC. Gastroenterology 2007;7:40 [Online publication].
10. The French, Metavir Cooperative study group. Intraobserver and interobserver variations in liver biopsy interpretation in patients with chronic hepatitis C. Hepatology 1994;20:15-20.

11. Castera L, Pinzani M. Biopsy and noninvasive methods for the diagnosis of liver fibrosis: Does it take two to tango? Gut 2010;59:861-66.

12. Forns X, Ampurdanes S, Llovet JM, et al. Identification of chronic hepatitis $\mathrm{C}$ patients without hepatic fibrosis by a simple predictive model. Hepatology 2002;36:986-92.

13. Wai CT, Greenson JK, Fontana RJ, et al. A simple noninvasive index can predict both significant fibrosis and cirrhosis in patients with chronic hepatitis C. Hepatology 2003;38:518-26.

14. Wu S, Wang J, Li L. Staging of liver fibrosis in chronic hepatitis B patients with a composite predictive model: A comparative study. World J Gastroenterol 2010;16:501-07.

15. Bedossa P, Poynard T. An algorithm for the grading of activity in chronic hepatitis C. The Metavir Cooperative study group. Hepatology 1996;24:289-93.

16. Williams AL, Hoofnagle JH. Ratio of serum aspartate to alanine aminotransferase in chronic hepatitis. Relationship to cirrhosis. Gastroenterology 1988;95:734-39.

17. Sebastiani G, Vario A, Guido M, Alberti A. Sequential algorithms combining noninvasive markers and biopsy for the assessment of liver fibrosis in chronic hepatitis B. World J Gastroenterol 2007;13:525-31.

18. Bedossa P, Dargère D, Paradis V. Sampling variability of liver fibrosis in chronic hepatitis C. Hepatology 2003;38:1449-57.

19. Murawaki Y, Ikuta Y, Okamoto K, Koda M, Kawasaki H. Diagnostic value of serum markers of connective tissue turnover for predicting histological staging and grading in patients with chronic hepatitis C. J Gastroenterol 2001;36:399-406.

20. Xu Y, DU WJ, Qin LY, Xing ZZ, Qin XH, Chen SJ. Expression of interleukin-17 in hepatitis B related liver fibrosis. Bao Xi, Fen Yu, Mianzi, Xue Yi, Zhi Za (Eds). 2009;25:133-35.

21. Murawaki Y, Yamada S, Ikuta Y, Kawasaki H. Clinical usefulness of serum matrix metalloproteinase-2 concentration in patients with chronic viral liver disease. J Hepatol 1999;30:1090-98.

22. Guéchot J, Laudat A, Loria A, Serfaty L, Poupon R, Giboudeau J. Diagnostic accuracy of hyaluronan and type III procollagen aminoterminal peptide serum assays as markers of liver fibrosis in chronic viral hepatitis $\mathrm{C}$ evaluated by ROC curve analysis. Clin Chem 1996;42:558-63.

23. Patel K, Lajoie A, Heaton S, et al. Clinical use of hyaluronic acid as a predictor of fibrosis change in hepatitis C. J Gastroenterol Hepatol 2003;18:253-57.

24. Montazeri G, Estakhri A, Mohamadnejad M, et al. Serum hyaluronate as a noninvasive marker of hepatic fibrosis and inflammation in HBeAg-negative chronic hepatitis B. BMC Gastroenterol 2005;5:32[Online publication].

25. Preaux AM, Mallat A, Van Nhieu JT, et al. Matrix metalloproteinase-2 activation in human hepatic fibrosis regulation by cell-matrix interactions. Hepatology 1999;30: 944-50.

26. Liu J, Shi BN, He JF. Effect of oxymatrine on serum matrix metalloproteinase-2 and its inhibitor in patients with chronic hepatitis B and liver cirrhosis. Zhong Zhongguo, Yi Xi, He Jie, Zhi Za (Eds) 2005;25:989-92.

27. Walsh KM, Timmus P, Campbell S, MacSween RN, Morris AJ. Plasma levels of matrix metalloproteinase-2 (MMP-2) and tissue inhibitors of metalloproteinase- 1 and 2 (TIMP-1 and TIMP-2) as noninvasive markers of liver disease in chronic hepatitis C: Comparison using ROC analysis. Dig Dis Sci 1999; 44:624-30. 
28. Boeker KH, Haberkorn CI, Michels D, Flemming P, Manns MP, Lichtinghagen R. Diagnostic potential of circulating TIMP1 and MMP-2 as markers of liver fibrosis in patients with chronic hepatitis C. Clin Chim Acta 2002;316:71-81.

29. Zhang Y, Wu W, Zhang Y, Feng Y, Zhou X, Pan Q. Noninvasive assessment of liver fibrosis with combined serum aminotransferase/platelet ratio index and hyaluronic acid in patients with chronic hepatitis B. World J Gastroenterol 2008;14:7117-21.
30. Peck-Radosavljevic M. Thrombocytopenia in liver disease. Can J Gastroenterol 2000;14:D60-D66.

31. Prati D, Taioli E, Zanella A, et al. Updated definitions of healthy ranges for serum alanine aminotransferase levels. Ann Intern Med 2002;137:1-10.

32. Bedossa P, Carrat F. Liver biopsy: The best, not the gold standard. J Hepatol 2009;50:1-3.

33. Mehta SH, Lau B, Afdhal NH, et al. Exceeding the limits of liver histology markers. J Hepatol 2009;50:36-41. 